\title{
Graphs on surfaces and Khovanov homology
}

\author{
ABHIJIT CHAMPANERKAR \\ ILYA KOFMAN \\ NeAL StOLTZFus
}

\begin{abstract}
Oriented ribbon graphs (dessins d'enfant) are graphs embedded in oriented surfaces. A quasi-tree of a ribbon graph is a spanning subgraph with one face, which is described by an ordered chord diagram. We show that for any link diagram $L$, there is an associated ribbon graph whose quasi-trees correspond bijectively to spanning trees of the graph obtained by checkerboard coloring $L$. This correspondence preserves the bigrading used for the spanning tree model of Khovanov homology, whose Euler characteristic is the Jones polynomial of $L$. Thus, Khovanov homology can be expressed in terms of ribbon graphs, with generators given by ordered chord diagrams.
\end{abstract}

57M25, 57M15; 05C10

In memory of Xiao-Song Lin

\section{Introduction}

A ribbon graph is a multi-graph (loops and multiple edges allowed) that is embedded in a surface, such that its complement is a union of 2-cells. In the case when the surface is oriented, the embedding determines a cyclic order on the edges at every vertex, which is called an orientation for the ribbon graph. Other terms for oriented ribbon graphs include: combinatorial maps, fat graphs, cyclic graphs, graphs with rotation systems, and dessins d'enfant (see Bollobás and Riordan [1]). In this paper, all ribbon graphs will be oriented.

The Jones polynomial of any link can be obtained as a specialization of the BollobásRiordan-Tutte polynomial of a ribbon graph obtained from the link diagram (see Dasbach, Futer, Kalfagianni, Lin and Stoltzfus [4]). The Jones polynomial also has an expansion in terms of spanning trees of the Tait graph, obtained by checkerboard coloring the link diagram. Moreover, with an appropriate bigrading, these spanning trees generate Khovanov homology, whose bigraded Euler characteristic is the Jones polynomial (see Champanerkar and Kofman [2]). 
We show that there is a one-to-one correspondence between spanning trees of the Tait graph and quasi-trees, which are spanning ribbon subgraphs with one face. We translate the data used to define the bigrading for spanning trees to the language of ribbon graphs using ordered chord diagrams. The Khovanov homology results in [2] are then expressed in terms of ribbon graphs and ordered chord diagrams. This leads to the question: Do any of the algebraic structures known for chord diagrams carry over to Khovanov homology?

This project was inspired by Dasbach, Futer, Kalfagianni, Lin and Stoltzfus [3] and Manturov [7], and the correspondence we establish implies some of their results. For example, we give a new proof that the thickness of Khovanov homology has a bound in terms of the Turaev genus.

The first author was supported by by National Science Foundation grant DMS-0455978, the second author by National Science Foundation grant DMS-0456227 and PSCCUNY grant 60046-3637, and the third author by National Science Foundation grant DMS-0456275.

\section{Quasi-trees and spanning trees}

Let $D$ be a connected link diagram. A checkerboard coloring of $D$ determines the Tait graph $G$. An edge of $G$ is positive if the shaded regions of its endpoints are joined by $A$-smoothing the corresponding crossing of $D$. Otherwise, the edge is negative. We take either $G$ or its planar dual so that $E_{+}(G) \geq E_{-}(G)$.

Let $\mathbb{G}$ be the all- $A$ ribbon graph of $D$ as defined in [4]. Let $V(\mathbb{G})$ be the number of vertices of $\mathbb{G}$, which is the number of components in the all- $A$ state of $D$. A ribbon subgraph $\mathbb{U} \subset \mathbb{G}$ is called a spanning subgraph if $V(\mathbb{M})=V(\mathbb{G})$. Let $F(\mathbb{M})$ be the number of faces of $\mathbb{H}$, which is the number of complementary regions in the orientable surface of minimal genus on which $\mathbb{H}$ embeds. The genus of this surface is called the

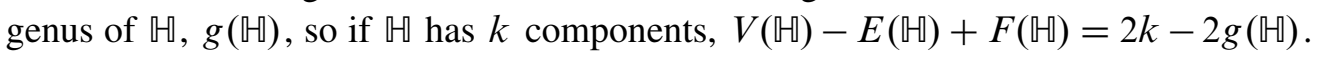
A quasi-tree $\mathbb{Q}$ is a spanning subgraph of $\mathbb{G}$ with $F(\mathbb{Q})=1$ (see [3, Definition 3.1]).

Theorem 2.1 Quasi-trees of $\mathbb{G}$ are in one-one correspondence with spanning trees of $G$ :

$$
\mathbb{Q}_{j} \leftrightarrow T_{v} \quad \text { where } \quad v+j=\frac{1}{2}\left(V(G)+E_{+}(G)-V(\mathbb{G})\right)
$$

$\mathbb{Q}_{j}$ denotes a quasi-tree of genus $j$, and $T_{v}$ denotes a spanning tree with $v$ positive edges. 
The proof will use the following lemma. Any state $s$ of $D$ corresponds to a unique map $\bar{s}$ : Edges $(\mathbb{G}) \rightarrow\{A, B\}$. Let $|s|$ denote the number of components in that smoothing of $D$. In $[4$, Section 4], the ribbon graph $\mathbb{G}(s)$ was defined such that $V(\mathbb{G}(s))=|s|$. We now define a different correspondence between states of $D$ and ribbon graphs:

Lemma 2.2 Spanning subgraphs $\mathbb{U} \subset \mathbb{G}$ are in one-one correspondence with states $s$ of $D$, such that $\bar{s}(\mathbb{H})(e)=B$ iff $e \in \mathbb{W}$. Thus, $F(\mathbb{M})=|s|$ and $V(\mathbb{H})=V(\mathbb{G})$.

Proof For any state $s$ of $D$, let $D_{s}$ denote the following link diagram:

$$
D_{s}= \begin{cases}A-\text { smoothing at } e & \text { if } \bar{s}(e)=A \\ D-\text { crossing at } e & \text { if } \bar{s}(e)=B\end{cases}
$$

Let $\mathbb{G}_{A}\left(D_{s}\right)$ and $\mathbb{G}_{B}\left(D_{s}\right)$ denote the all- $A$ and all- $B$ ribbon graphs of $D_{s}$, respectively. For any state $s$ of $D$, define $\mathbb{H}(s)=\mathbb{G}_{A}\left(D_{S}\right)$. If we identify $s$ with $\bar{s}$, then $s=\bar{s}(\mathbb{W}(s))$ and $\mathbb{W}=\mathbb{U}(\bar{s}(\mathbb{M}))$.

The ribbon graphs $\mathbb{H}(s)=\mathbb{G}_{A}\left(D_{s}\right)$ and $\mathbb{G}_{B}\left(D_{s}\right)$ are dual in the sense of Dasbach, Futer, Kalfagianni, Lin and Stoltzfus [4, Lemma 4.1]. By the duality, $F(\mathbb{H}(s))=$ $V\left(\mathbb{G}_{B}\left(D_{s}\right)\right)=|s|$. Also, $V(\mathbb{H}(s))=V\left(\mathbb{G}_{A}\left(D_{s}\right)\right)=V(\mathbb{G})$.

A Jordan trail of a connected link diagram is a choice of smoothings at each crossing that results in a simple closed curve (see Kauffman [5, page 2]). There is a one-one correspondence between Jordan trails of $D$ and spanning trees of the Tait graph $G$ [5, page 56]. In particular, the Jordan trail of a spanning tree $T$ bounds a planar neighborhood of $T$.

Proof of Theorem 2.1 In the table below, let $\tau, t, \bar{\tau}, \bar{t}$ denote a positive edge in $T$, a positive edge in $G-T$, a negative edge in $T$, and a negative edge in $G-T$, respectively. The Jordan trail of $T$ is then given by the smoothings of $D$ shown in the second row. By Lemma 2.2, each Jordan trail corresponds to a spanning subgraph of $\mathbb{G}$ with one face, which is a quasi-tree. Let $\mathbb{Q}$ be the quasi-tree that corresponds to $T$. If $Q \in \mathbb{Q}$ and $q \in(\mathbb{G}-\mathbb{Q})$, then $s(\mathbb{Q})$, given in Lemma 2.2, determines the correspondence:

$$
\begin{array}{l|l|l|l}
\tau & t & \bar{\tau} & \bar{t} \\
A & B & B & A \\
q & Q & Q & q
\end{array}
$$

To prove the numerical claim, for any $T$ in $G, v(T)=\# \tau$ and $E(\mathbb{Q})=\# t+\# \bar{\tau}$.

$$
\begin{aligned}
j & =\frac{1}{2}(1-V(\mathbb{Q})+E(\mathbb{Q}))=\frac{1}{2}(1-V(\mathbb{G})+\# t+\# \bar{\tau}) \\
v+j & =\frac{1}{2}(2(\# \tau)+1-V(\mathbb{G})+\# t+\# \bar{\tau})=\frac{1}{2}\left(V(G)+E_{+}(G)-V(\mathbb{G})\right)
\end{aligned}
$$

since $\# \tau+\# \bar{\tau}=E(T)=V(G)-1$ and $\# \tau+\# \bar{t}=E_{+}(G)$. 


\section{Quasi-tree complex for Khovanov homology}

To construct the spanning tree chain complex in Champanerkar-Kofman [2], every spanning tree $T$ of the Tait graph $G$ was given a bigrading $(u(T), v(T))$. By Theorem 2.1 , the $v$-grading, which is the number of positive edges in $T$, is determined by the genus of the corresponding quasi-tree $\mathbb{Q}$. The $u$-grading, which was defined using activities in the sense of Tutte, also has a quasi-tree analogue in terms of the ordered chord diagram for $\mathbb{Q}$.

If $D$ has $n$ ordered crossings, let $\mathbb{G}$ be given by permutations $\left(\sigma_{0}, \sigma_{1}, \sigma_{2}\right)$ of the set $\{1, \ldots, 2 n\}$, such that the $i$ th crossing corresponds to half-edges $\{2 i-1,2 i\}$, which are marked on the components of the all- $A$ state of $D$. For an example, see Figure 2 in Section 4. We give the components of the all $-A$ state of $D$ the admissible orientation for which outer ones are oriented counterclockwise (see Dasbach, Futer, Kalfagianni, Lin and Stoltzfus [4]). In this way, every component has a well-defined positive direction.

The orbits of $\sigma_{0}$ form the vertex set. In particular, $\sigma_{0}$ is given by noting the half-edge marks when going in the positive direction around the components of the all- $A$ state of $D$. The other permutations are given by $\sigma_{1}=\prod_{i=1}^{n}(2 i-1,2 i)$ and $\sigma_{2}=\sigma_{1} \circ \sigma_{0}^{-1}$

Let an ordered chord diagram denote a circle marked with $\{1, \ldots, 2 n\}$ in some order, and chords joining all pairs $\{2 i-1,2 i\}$.

Proposition 3.1 Every quasi-tree $\mathbb{Q}$ corresponds to the ordered chord diagram $C_{\mathbb{Q}}$ with consecutive markings in the positive direction given by the permutation:

$$
\sigma(i)= \begin{cases}\sigma_{0}(i) & i \notin \mathbb{Q} \\ \sigma_{2}^{-1}(i) & i \in \mathbb{Q}\end{cases}
$$

Proof Since $\mathbb{Q}$ is a quasi-tree, $\gamma_{\mathbb{Q}}$ is one simple closed curve. If we choose an orientation on $S(\mathbb{G})$, we can traverse $\gamma_{\mathbb{Q}}$ along successive boundaries of bands and vertex discs, such that we always travel around the boundary of each disc in a positive direction (i.e., the disc is on the left). If a half-edge is not in $\mathbb{Q}, \gamma_{\mathbb{Q}}$ will pass across it travelling along the boundary of a vertex disc to the next band. If a half-edge is in $\mathbb{Q}, \gamma_{\mathbb{Q}}$ traverses along one of the edges of its band. On $\gamma_{\mathbb{Q}}$, we mark a half-edge not in $\mathbb{Q}$ when $\gamma_{\mathbb{Q}}$ passes across it along the boundary of the vertex disc and we mark a half-edge in $\mathbb{Q}$ when we traverse an edge of a band in the direction of the half-edge. If the half-edge $i$ is not in $\mathbb{Q}$, travelling along the boundary of a vertex disc, the next half-edge is given by $\sigma_{0}$. If the half-edge $i$ is in $\mathbb{Q}$, traversing the edge of its band to 

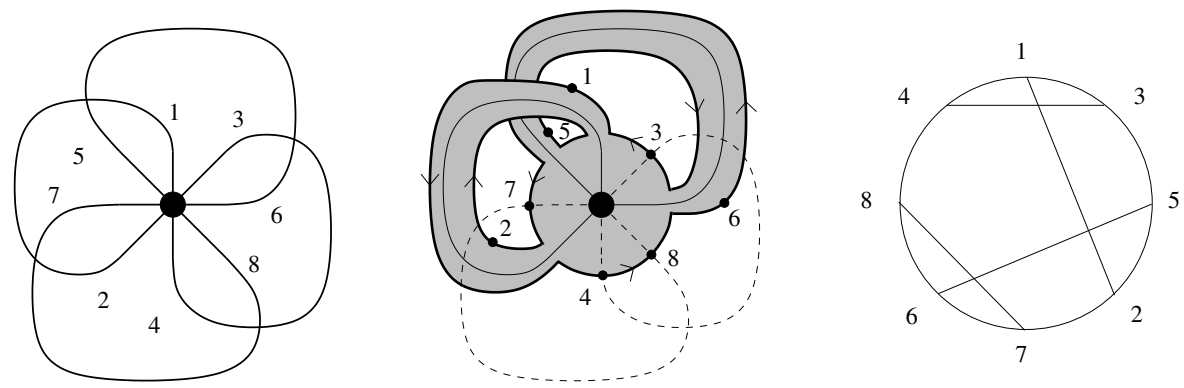

Figure 1: Ribbon graph $\mathbb{G}$, quasi-tree $\mathbb{Q}=(12)(56)$ with curve $\gamma_{\mathbb{Q}}$, chord diagram $C_{\mathbb{Q}}$

the vertex disc and then along the boundary of that disc, the next half-edge is given by $\sigma_{0} \sigma_{1}=\sigma_{2}^{-1}$. For example, see Figure 1 .

As $\mathbb{Q}$ is a quasi-tree, each of its half-edges must be in the orbit of its single face, while the complementary set of half-edges are met along the boundaries of the vertex discs. As we mark all half-edges traversing $\gamma_{\mathbb{Q}}$, the chord diagram $C_{\mathbb{Q}}$ parametrizes $\gamma_{\mathbb{Q}}$.

Note that if $\mathbb{G}$ is the all- $A$ ribbon graph of a connected link diagram $D$, by the proof of Theorem 2.1, following $\gamma_{\mathbb{Q}}$ along an edge of $\mathbb{Q}$ is given by the $B$-smoothing of that crossing of $D_{s(\mathbb{Q})}$. Therefore, the chord diagram $C_{\mathbb{Q}}$ parametrizes both $\gamma_{\mathbb{Q}}$ and the Jordan trail for $T$, which is the all- $B$ state of $D_{s(\mathbb{Q})}$.

To compute the genus $g(\mathbb{Q})$ from $C_{\mathbb{Q}}$, let $C$ be the sub-chord diagram of chords that correspond to edges in $\mathbb{Q}$. Then $g(\mathbb{Q})$ is half the rank of the adjacency matrix of the intersection graph of $C$ (see Bollobás and Riordan [1]).

Definition 3.1 Using $\min \left(i, \sigma_{1}(i)\right)$, there is an induced total order on the chords of $C_{\mathbb{Q}}$. A chord is live if it does not intersect lower-ordered chords, and otherwise it is dead. For any quasi-tree $\mathbb{Q}$, an edge $e$ is live or dead when the corresponding chord of $C_{\mathbb{Q}}$ is live or dead.

In Figure 1, we show $C_{\mathbb{Q}}$ such that the only edge live with respect to $\mathbb{Q}$ is (12).

For every spanning tree $T$ of $G$, each edge $e \in G$ has an activity with respect to $T$, as originally defined by Tutte. If $e \in T, \operatorname{cut}(T, e)$ is the set of edges that connect $T \backslash e$. If $f \notin T, \operatorname{cyc}(T, f)$ is the set of edges in the unique cycle of $T \cup f$. Note $f \in \operatorname{cut}(T, e)$ if and only if $e \in \operatorname{cyc}(T, f)$. An edge $e \in T$ (resp. $e \notin T$ ) is live if it is the lowest edge in its cut (resp. cycle), and otherwise it is dead.

Lemma 3.2 If $T$ corresponds to $\mathbb{Q}$, as in Theorem 2.1, then the $i$ th edge of $\mathbb{G}$ is live with respect to $\mathbb{Q}$ if and only if the $i$ th edge of $G$ is live with respect to $T$. 
Proof In $C_{\mathbb{Q}}$, the $i$ th and $j$ th chords intersect if and only if going around the Jordan trail for $T$ in some direction, we see cyclic permutations of the marks $(2 i-1,2 j-$ $1,2 i, 2 j)$ or $(2 i-1,2 j, 2 i, 2 j-1)$. Now, $e_{i} \in \operatorname{cut}\left(T, e_{j}\right)$ or $e_{i} \in \operatorname{cyc}\left(T, e_{j}\right)$ if and only if the Jordan trail becomes disconnected when the $j$ th smoothing is changed, and is re-connected when the $i$ th smoothing is changed (see Kauffman [6]). Equivalently, $C_{\mathbb{Q}}$ becomes disconnected when unzipped along the $j$ th chord, and becomes re-connected when unzipped along the $i$ th chord, which occurs if and only if the $i$ th and $j$ th chords intersect:

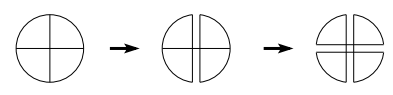

Therefore, $e_{i}$ is live with respect to $T$ exactly when the $i$ th chord does not intersect lower-ordered chords.

Definition 3.3 For any quasi-tree $\mathbb{Q}$ of $\mathbb{G}$, we define

$$
u(\mathbb{Q})=\#\{\text { live edges not in } \mathbb{Q}\}-\#\{\text { live edges in } \mathbb{Q}\} \quad \text { and } \quad v(\mathbb{Q})=-g(\mathbb{Q})
$$

Define $\mathcal{C}(\mathbb{G})=\oplus_{u, v} \mathcal{C}_{v}^{u}(\mathbb{G})$, where $\mathcal{C}_{v}^{u}(\mathbb{G})=\mathbb{Z}\langle\mathbb{Q} \subset \mathbb{G} \mid u(\mathbb{Q})=u, v(\mathbb{Q})=v\rangle$

Theorem 3.4 For a knot diagram $D$, there exists a quasi-tree complex $\mathcal{C}(\mathbb{G})=$ $\left\{\mathcal{C}_{v}^{u}(\mathbb{G}), \partial\right\}$ with $\partial: \mathcal{C}_{v}^{u} \rightarrow \mathcal{C}_{v-1}^{u-1}$ that is a deformation retract of the reduced Khovanov complex. In particular, the reduced Khovanov homology $\widetilde{H}^{i, j}(D ; \mathbb{Z})$ is given by

$$
\widetilde{H}^{i, j}(D ; \mathbb{Z}) \cong H_{v}^{u}(\mathcal{C}(\mathbb{G}) ; \mathbb{Z})
$$

with the indices related as follows:

$$
u=j-i-w(D)+1 \quad \text { and } \quad v=j / 2-i+\left(V(\mathbb{G})-c_{+}(D)\right) / 2
$$

where $w(D)$ is the writhe, $c_{+}(D)$ is the number of positive crossings of $D$, and $V(\mathbb{G})$ is the number of components in the all- $A$ state of $D$, which is the number of vertices of $\mathbb{G}$.

Proof The result follows from Champanerkar-Kofman [2, Theorem 5] and Theorem 2.1 , once we establish for a spanning tree $T$ corresponding to a quasi-tree $\mathbb{Q}$ that their bigradings are related as claimed.

By Lemma 3.2, edges of $\mathbb{Q}$ and $T$ are live exactly when they correspond. From [2], $u(T)=\# L-\# \ell-\# \bar{L}+\# \bar{\ell}$. By the proof of Theorem 2.1, $\{L, \bar{\ell}\}$-edges of $T$ 
correspond to live edges not in $\mathbb{Q}$, and $\{\ell, \bar{L}\}$-edges of $T$ correspond to live edges in $\mathbb{Q}$. Therefore,

$$
u(\mathbb{Q})=\#\{\text { live edges not in } \mathbb{Q}\}-\#\{\text { live edges in } \mathbb{Q}\}=u(T)=j-i-w(D)+1
$$

By [2, Theorem 5] and Theorem 2.1,

$$
\begin{aligned}
v(\mathbb{Q}) & =-g(\mathbb{Q})=v(T)-\frac{V(G)+E_{+}(G)-V(\mathbb{G})}{2} \\
& =\left(\frac{j}{2}-i-\frac{w(D)-k(D)-2}{4}\right)-\frac{V(G)+E_{+}(G)-V(\mathbb{G})}{2} \\
& =\frac{j}{2}-i-\frac{w(D)+E(G)}{4}+\frac{V(\mathbb{G})}{2}=\frac{j}{2}-i+\frac{V(\mathbb{G})-c_{+}(D)}{2}
\end{aligned}
$$

where we used that $w(D)+E(G)=w(D)+c(D)=2 c_{+}(D)$.

Remark Similarly, for any knot diagram $D$ there exists a quasi-tree complex that is a deformation retract of the (unreduced) Khovanov complex. Let $\overline{\mathcal{C}}_{v+1}^{u+2}(\mathbb{G})=\mathcal{C}_{v}^{u}(\mathbb{G})$. Let $\mathcal{U C} \mathcal{C}_{v}^{u}(\mathbb{G})=\left(\mathcal{C}_{v}^{u}(\mathbb{G})+\overline{\mathcal{C}}_{v+1}^{u+2}(\mathbb{G}), \partial\right)$. By [2, Theorem 6] and the rest of the proof of Theorem $3.4, H^{i, j}(D ; \mathbb{Z}) \cong H_{v}^{u}(\mathcal{U C}(\mathbb{G}) ; \mathbb{Z})$, with the indices related as above.

Let $\mathbb{G}(D)$ be the all- $A$ ribbon graph of a connected link diagram $D$. As defined by Dasbach, Futer, Kalfagianni, Lin and Stoltzfus [4], for any link L, the Turaev genus $g_{T}(L)$ is the minimum value of $g(\mathbb{G}(D))$ among all connected diagrams $D$ of $L$. The following bound for the thickness of Khovanov homology in terms of Turaev genus was obtained by Manturov [7]:

Corollary 3.1 For any knot $K$ with Turaev genus $g_{T}(K)$, the thickness of the reduced Khovanov homology of $K$ is less than or equal to $g_{T}(K)+1$. The thickness of the (unreduced) Khovanov homology of $K$ is less than or equal to $g_{T}(K)+2$.

Proof Let $D$ be any diagram of $K$ and $\mathbb{G}$ its all- $A$ ribbon graph. For any quasi-tree $\mathbb{Q}$ of $\mathbb{G},-g(\mathbb{G}) \leq v(\mathbb{Q}) \leq 0$. Therefore, $\mathcal{C}(\mathbb{G})$ has $g(\mathbb{G})+1$ rows, so $H_{v}^{u}(\mathcal{C}(\mathbb{G}) ; \mathbb{Z})$ has at most $g(\mathbb{G})+1$ rows. Similarly, by the Remark above, $H_{v}^{u}(\mathcal{U C}(\mathbb{G}) ; \mathbb{Z})$ has at most $g(\mathbb{G})+2$ rows. Hence the bound follows from the definition of Turaev genus.

Corollary 3.1 is stronger than [2, Theorem 13(ii)] because, for instance, knots with Turaev genus one are a much richer class than 1-almost alternating knots. (For example, see Dasbach, Futer, Kalfagianni, Lin and Stoltzfus [3, Lemma 4.3].) However, by the correspondence in Theorem 2.1, the two proofs are the same: Since $0 \leq j \leq g(\mathbb{G})$,

$$
g(\mathbb{G})=\max _{T \subset G} v(T)-\min _{T \subset G} v(T)
$$


Corollary 3.2 The Turaev genus of $(3, q)$-torus knots is unbounded.

Proof The rational Khovanov homology of $(3, q)$-torus links was computed by Turner [8]. From this work, the thickness of the Khovanov homology of torus knots of type $(3,3 N+1)$ and $(3,3 N+2)$ is exactly $N+2$. By Corollary 3.1 , the Turaev genus of these torus knots is at least $N$.

\section{Example}

As an example, we use a 4-crossing diagram of the trefoil. In Figure 2, we show the diagram $D$, the Tait graph $G$, the all- $A$ ribbon graph $\mathbb{G}$ and its surface, given by $\sigma_{0}=(15724863), \sigma_{1}=(12)(34)(56)(78)$ and $\sigma_{2}=(14)(2835)(67)$.

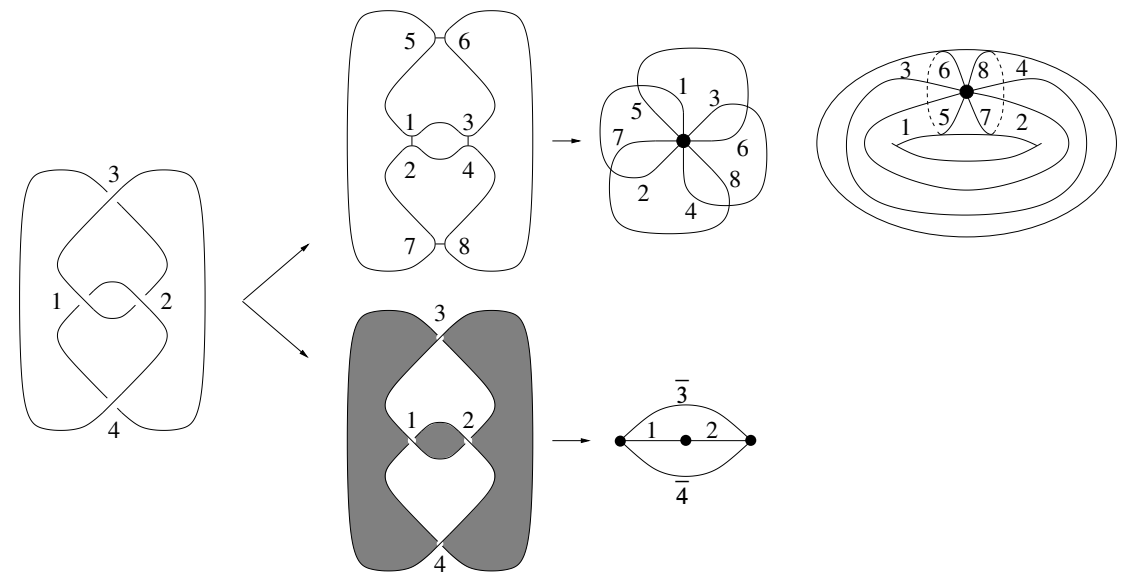

Figure 2: The 4-crossing trefoil, the Tait graph $G$, the all- $A$ ribbon graph $\mathbb{G}$ and its surface

The ordered chord diagram for each quasi-tree is given by Proposition 3.1. This order can be seen from the corresponding Jordan trail, which is shown for the quasi-tree $\mathbb{Q}_{1}$ with edges (12) and (56):

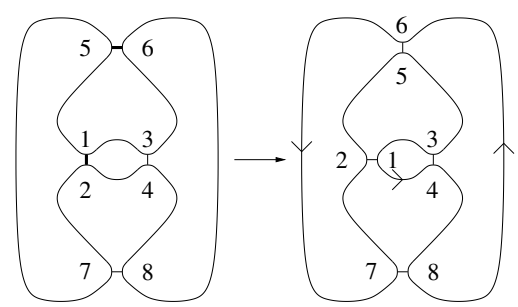

Algebraic 83 Geometric Topology, Volume 7 (2007) 
Below we show the correspondence between the spanning trees of $G$ and the chord diagrams for the quasi-trees of $\mathbb{G}$. The circled numbers indicate edges in $\mathbb{G}-\mathbb{Q}$. The activities follow the convention: capital letters for edges in the spanning tree or quasi-tree, bar for negative edges, $L$ or $\ell$ for live, $D$ or $d$ for dead.

\begin{tabular}{|l|l|l|l|l|}
\hline $\mathbb{Q}_{1}=L d D d$ & $\mathbb{Q}_{2}=L d \ell D$ & $\mathbb{Q}_{3}=\ell D D d$ & $\mathbb{Q}_{4}=\ell D \ell D$ & $\mathbb{Q}_{5}=\ell \ell d$ \\
\hline$T_{1}=\ell D \bar{D} \bar{d}$ & $T_{2}=\ell D \bar{\ell} \bar{D}$ & $T_{3}=L d \bar{D} \bar{d}$ & $T_{4}=L d \bar{\ell} \bar{D}$ & $T_{5}=L L \bar{d} \bar{d}$ \\
\hline
\end{tabular}

\section{References}

[1] B Bollobás, O Riordan, A polynomial invariant of graphs on orientable surfaces, Proc. London Math. Soc. (3) 83 (2001) 513-531 MR1851080

[2] A Champanerkar, I Kofman, Spanning trees and Khovanov homology arXiv: math.GT/0607510

[3] O Dasbach, D Futer, E Kalfagianni, X-S Lin, N Stoltzfus, Alternating sum formulae for the determinant and other link invariants arXiv:math.GT/0611025

[4] O Dasbach, D Futer, E Kalfagianni, X-S Lin, N Stoltzfus, The Jones polynomial and graphs on surfaces arXiv:math.GT/0605571

[5] L H Kauffman, Formal knot theory, Mathematical Notes 30, Princeton University Press, Princeton, NJ (1983) MR712133

[6] L H Kauffman, A Tutte polynomial for signed graphs, Discrete Appl. Math. 25 (1989) 105-127 MR1031266

[7] V Manturov, Minimal diagrams of classical and virtual links arXiv: math.GT/0501393

[8] P Turner, A spectral sequence for Khovanov homology with an application to $(3, q)-$ torus links arXiv:math.GT/0606369 
Department of Mathematics and Statistics, University of South Alabama

Mobile AL 36688, USA

Department of Mathematics, College of Staten Island, City University of New York 2800 Victory Boulevard, Staten Island NY 10314, USA

Department of Mathematics, Louisiana State University

Baton Rouge LA 70803-4918, USA

achampanerkar@jaguar1.usouthal.edu, ikofman@math.csi.cuny.edu, stoltz@math.lsu.edu

http://(www.southalabama.edu/mathstat/personal_pages/achamp/, http://www . math.csi.cuny. edu/ ikofman/,

http://www.math.lsu.edu/ stoltz/

Received: 27 May 2007

Algebraic 8 Geometric $\mathcal{T}$ opology, Volume 7 (2007) 